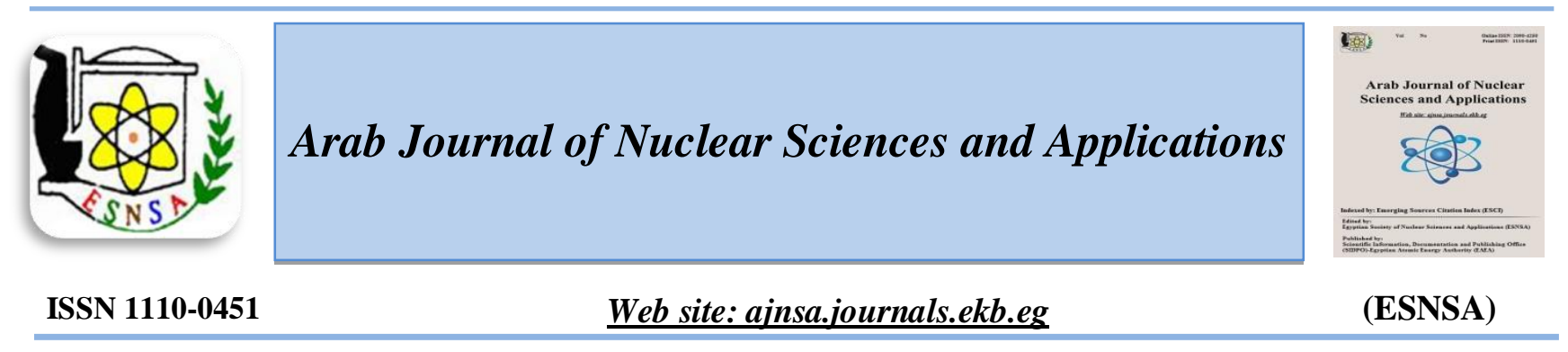

\title{
Electrical Characteristics, Plasma parameters and Reaction Rates of $\mathrm{DC} \mathrm{O}_{2}$ Plasma
}

\author{
O. F. Farag \\ Zagazig University, Faculty of Science, Physics Department, Zagazig, Egypt
}

The electrical characteristics of DC oxygen $\left(\mathrm{O}_{2}\right)$ plasma such as the discharge current-voltage (I-V) curves and the breakdown voltage of oxygen gas were measured. The plasma parameters such as electron temperature $\left(T_{e}\right)$ and density $\left(n_{e}\right)$ were obtained using Langmuir probe at a constant power over a wide rangeof $\mathrm{O}_{2}$ gas pressures. The measured $T_{e}$ values were used to calculate the various rate coefficients for electron impact (ionization, dissociation and excitation) from ground state of $\mathrm{O}_{2}$ molecules. Thereafter, the calculated rate coefficients and the measured $n_{e}$ values were used to calculate the rates of ionization, dissociation and excitation from ground state of $\mathrm{O}_{2}$ molecules. The I-V measurements of the discharge and Paschen curve show that the operating voltage decreased with increasing $\mathrm{O}_{2}$ gas pressures at constant current and the minimum of Paschen curve was found to be 400 $\mathrm{V}$ at $\mathrm{Pd}=\mathbf{1 . 8 6}$ mbar.cm, respectively. The $T_{e}$ showed a decreasing trend with increasing $\mathrm{O}_{2}$ gas pressures while $n_{e}$ showed an increasing trend. The rate coefficients of ionization, dissociation and excitation were found to decrease with increasing $\mathrm{O}_{2}$ gas pressure. On the contrary, the rates of ionization, dissociation and excitation were found to increase with increasing $\mathrm{O}_{2}$ gas pressure.

Keywords: DC plasma / Electron temperature / Electron density / Rate coefficients / Ionization and dissociation rates

\section{Introduction}

Low pressure oxygen plasmas have been used in the field of plasma processing for decades with different applications such as ashing of photoresist [1], oxidation or thin film oxides deposition and removing organic polymide films [2,3].Oxygen discharge plasmas have also been used in the sterilization processes (e.g. bacteria inactivation) and improving surface properties of polymeric materials [4,5]. Low pressure oxygen discharge plasma is an electronegative plasma consisting of free electrons, positive ions, and negative ions, which were produced by the electron attachment to the neutral atoms or molecules [6]. Low pressure oxygen discharge plasma can be generated using several types of energy sources such as microwave (MW), radiofrequency (RF) and direct high voltage (DC HV). Although the plasma density produced by applying RF and MW as energy source is higher than that produced by applying of DC HV.The DC plasma has several advantages compared to the other energy sources. It doesnot require a matching unit and it is of the lowest cost. Moreover, the DC plasma reactors have the advantages of low maintenance cost and simple operation. Depending on the operating voltage and current the DC plasma are divided into several types such as the Townsend or dark, glow and arc discharges [7].

Oxygen plasmas contain several reactive species such as metastable oxygen molecules $\mathrm{O}_{2}\left(a^{1} \Delta_{g}, \mathrm{~b}\right.$ $\left.{ }^{1} \Sigma_{g}^{+}\right), \mathrm{O}^{+}{ }_{2}$ and oxygen atoms $\mathrm{O}\left({ }^{3} \mathrm{P},{ }^{1} \mathrm{D}\right)$, which renders it the best in many applications [8]. The previous reactive species are produced due to

Corresponding author: faragfarag66@yahoo.com

DOI: 10.21608/ajnsa.2019.6818.1161

(C) Scientific Information, Documentation and Publishing Office (SIDPO)-EAEA 
ionization, excitation and dissociation processes of neutral species in these plasmas. Therefore, it is necessary to study and understand the rate coefficients and rates of the processes responsible for the production of these active species. The electron temperature is an important parameter for understanding the rate coefficients of ionization, excitation and dissociation in oxygen plasmas.

In the present work, $\mathrm{DC} \mathrm{O}_{2}$ plasmas system was characterized. The electrical characteristics of the system such as the breakdown voltage in terms of Paschen curve and the I-V curves were evaluated and discussed in detail. The single Langmuir probe was used to determine plasma parameters, such as electron temperature $\left(\mathrm{T}_{\mathrm{e}}\right)$ and electron density $\left(\mathrm{n}_{\mathrm{e}}\right)$. The rate coefficients of the most important processes occurring in oxygen plasmas were evaluated using the values of electron temperature $\left(T_{e}\right)$,then, the values of rate coefficients are used in the evaluation of rate of electron impact ionization, dissociation and excitation.

\section{Material and Method}

A schematic diagram of the DC oxygen plasma experimental set up is shown in Fig.(1). The set-up of the system includes the following components. Plasma chamber, DC power supply, Vacuum system and Langmuir single probe. The plasma chamber is made of a Pyrex glass cylinder of 13 $\mathrm{cm}$ inner diameter and $20 \mathrm{~cm}$ in length. Two parallel movable stainless-steel discsof $100 \mathrm{~mm}$ diameter acted as discharge electrodes. The inter- electrode separation is adjusted to be $70 \mathrm{~mm}$. The low pressure in the plasma chamber is achieved by using a rotary pump (Edwards H. vacuum pump, model ED 200) which can evacuate the chamber pressure down to 0.0013 mbar. The flow of $\mathrm{O}_{2}$ is monitored with mass flow controller and enters the plasma chamber via controllable needle valve whereas the working pressure in the plasma chamber is monitored by using pirani gauge. The low pressure $\mathrm{O}_{2}$ plasmas is ignited by DC power supply which can produce discharge voltage up to $2 \mathrm{~K} \mathrm{~V}$.

In order to get the required data for plotting the breakdown (Paschen) curves, the inter-electrode separation was fixed at $70 \mathrm{~mm}$ while the working gas pressure was adjusted from 0.053 to 1.33 mbar. On the other hand, the I-V characteristic curves of the discharge are obtained by varying the potential difference between the two electrodes and recording the corresponding current in the pressure range from 0.133 to 1.33 mbar. Finally, the internal plasma parameters such as $T_{e}$ and $n_{e}$, which are required to understand the rate coefficients of ionization, excitation and dissociation in oxygen plasmas were obtained by using single Langmuir probe. The Langmuir probe is a Tungsten wire enclosed in a Pyrex glass tube. The probe tip is of $0.03 \mathrm{~cm}$ diameter and $0.5 \mathrm{~cm}$ length. The I-V characteristics of single Langmuir Probe were plotted by recording the probe current for a $\mathrm{DC}$ bias voltages ranging from -50 to $+50 \mathrm{~V}$.
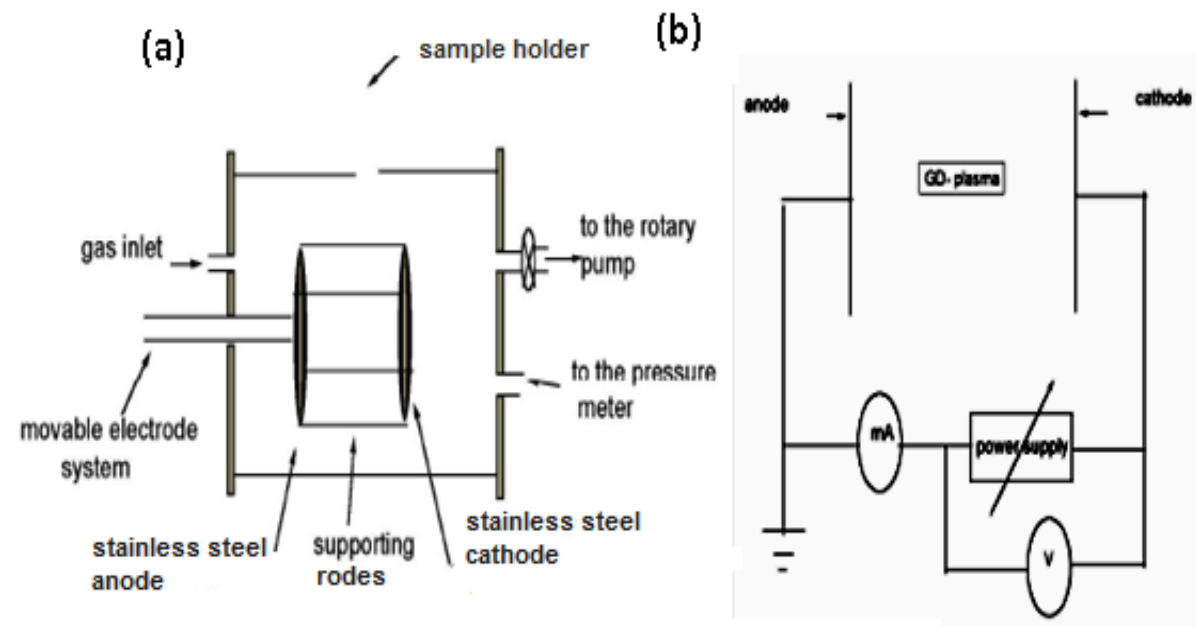

Fig. (1): A schematic dagram for (a) The experimental equipment and (b) The electrical circuit 


\section{Results and Discussion}

\section{Electrical Characteristics}

The dependence of the breakdown voltage $\left(\mathrm{V}_{\mathrm{B}}\right)$ on the product of working gas pressure $(\mathrm{P})$ and the inter electrode separation (d) is usually represented by a Paschen curve. The measurements of breakdown voltage values were determined as a function of $(\mathrm{Pd})$, where inter electrode separation was fixed at $7 \mathrm{~cm}$ and the $\mathrm{O}_{2}$ gas pressure varied from 0.053 to 1.33 mbar and the results are shown in Fig.(2). The Pd parameter is a scaling parameter proportional to the frequency of electron-neutral collisions over unit distance. Accordingly, atypical sudden increase of $\mathrm{V}_{\mathrm{B}}$ at low Pd-s can be attributed to the small number of electron-neutral oscillation frequencyFig.(2). On the other hand, at high Pd-s $\mathrm{V}_{\mathrm{B}}$ is increased slowly due to a large number of electron-neutral oscillation frequency. There is a minimum value between low and high $\mathrm{Pd}-\mathrm{s}$, at which the generation of charges by electron impact ionization and secondary electron emission mechanisms and losses by drift, diffusion and attachment are balanced [9]. Our minimum breakdown voltage is $400 \mathrm{~V}$ at $\mathrm{Pd}=1.86$ (mbar.cm) and this value in agreement with reported by Khalaf et al. [10].

The current-volt (I-V) characteristic curves at different working pressures of $\mathrm{O}_{2}$ gas are shown in Fig.(3). From this figure It can be noticed that as the applied voltage increase, the discharge current increase at the same $\mathrm{O}_{2}$ gas pressure.Moreover, it is clearly seen that at the same discharge current, the applied voltage decreases with increasing the $\mathrm{O}_{2}$ gas pressure. At a discharge current of $3 \mathrm{~mA}$, the applied voltage decreases from $702 \mathrm{~V}$ to $450 \mathrm{~V}$ as the $\mathrm{O}_{2}$ gas pressure increases from 0.133 to 1.33 mbar. In other words, plasma electrical conductivity increases by increasing the $\mathrm{O}_{2}$ gas pressure. In plasma, both ions and electrons are carrying the electrical plasma current. The plasma electrical conductivity is given by the microscopic plasma parameters [11]:

$\sigma=\frac{e^{2} n_{e}}{m_{e} v}$

Where e and $m_{e}$ are the electron electric charge and mass, respectively, $\mathrm{n}_{\mathrm{e}}$ is the plasma electron number density and $v$ is the frequency of collisions between electrons and neutral atoms or molecules in plasma.

With increasing the $\mathrm{O}_{2}$ gas pressure in the plasma chamber the electron number density is increased. This is also observed from the single Langmuir probe measurements which will be shown later. The behavior of applied voltage with $\mathrm{O}_{2}$ gas pressure at constant discharge current is similar to that reported by K. Yasserian et al. and O. F. Farag $[12,13]$.

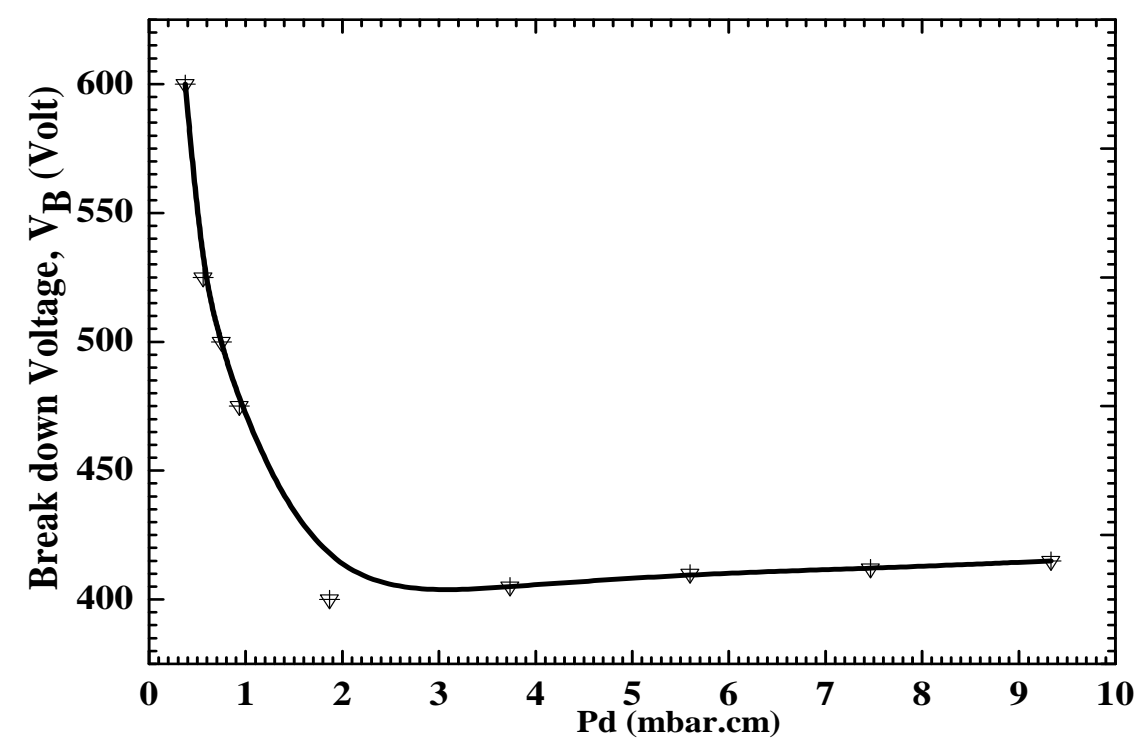

Figure (2): Paschen curve show the variation of $V_{B}$ with the product $P d$ of $\mathrm{O}_{2}$ at $\mathrm{d}=7 \mathrm{~cm}$ 


\section{Plasma Parameters}

The electron temperature, $T_{e}$, and electron number density, $\mathrm{n}_{\mathrm{e}}$, are calculated from single Langmuir probe I-V characteristic curve by using the appropriate expressions as shown in Mansour et al. (2013) and Farag and Mansour (2013) [14,15]. The influence of working gas pressure on $T_{e}$ and $n_{e}$ was discussed in detail. First, the influence of working gas pressure on the $T_{e}$ and $n_{e}$ was investigated while the power of $\mathrm{O}_{2}$ discharge is adjusted at about 3.5 W. As illustrated in Fig.(4), $\mathrm{T}_{\mathrm{e}}$ decreases with the increase in $\mathrm{O}_{2}$ gas pressure inside the plasma chamber at a constant discharge power. This is in contrast to the behavior of $n_{e}$, which increased with increasing $\mathrm{O}_{2}$ gas pressure. The values of $\mathrm{T}_{\mathrm{e}}$ and $\mathrm{n}_{\mathrm{e}}$ are about $6.69 \mathrm{eV}$ and $0.7258 \times$ $10^{9} \mathrm{~cm}^{-3}$ at $\mathrm{O}_{2}$ gas pressure and applied power are about 0.133 mbar and $3.5 \mathrm{~W}$, respectively. As the $\mathrm{O}_{2}$ gas pressure increases to 1.06 mbar and the applied power remains constant, $\mathrm{T}_{\mathrm{e}}$ decreases to $4.27 \mathrm{eV}$ and $\mathrm{n}_{\mathrm{e}}$ increases to $5.304 \times 10^{9} \mathrm{~cm}^{-3}$ as shown in Fig. (4). The behavior of $T_{e}$ and $n_{e}$ with $\mathrm{O}_{2}$ gas pressure is consistent with that reported by Gudmundsson et al. (2000) [16].

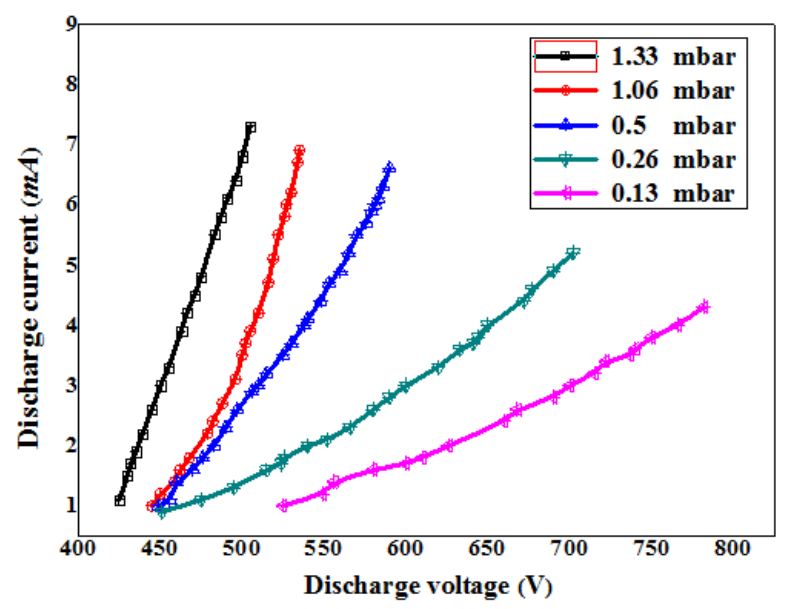

Figure (3): The I-V characteristic curves of $\mathrm{O}_{2}$ at different working pressures (chamber pressure) at distance $7 \mathrm{~cm}$

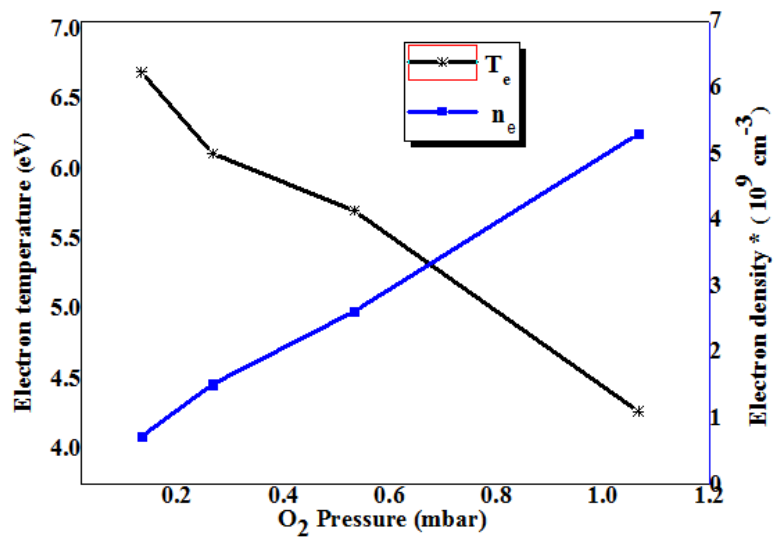

Figure (4): Electron (temperature and density) variation with $\mathrm{O}_{2}$ gas pressure at discharge power $3.5 \mathrm{~W}$

The axial profile of $T_{e}$ and $n_{e}$ for oxygen discharge at operating conditions of $\mathrm{O}_{2}$ gas pressure $=0.5$ mbar and applied power about $3.5 \mathrm{~W}$ is shown in Figure 5. The value of $T_{e}$ is highest at the edge of the cathode fall region (CF). This may be attributed to two reasons, first the high electric field and second, the absence of inelastic collisions between the accelerated electrons with oxygen molecules and/or atoms in this region. Beyond the $\mathrm{CF}$ region, the value of $\mathrm{T}_{\mathrm{e}}$ drops through the negative glow region (where the electric field drops in this region and many ionization and excitation collisions occur) and becomes nearly constant throughout the whole region to the anode (also this could be attributed to the very weak electric field). From Figure 5 the axial profile of $n_{e}$ can be described as, the value of $n_{e}$ is the lowest in the $\mathrm{CF}$ region and in the negative glow region it reached maximum value. Moreover, the values of $\mathrm{n}_{\mathrm{e}}$ are nearly constant through the negative glow region and near the anode region dropping slightly. The axial profiles of $T_{e}$ and $n_{e}$ are similar to that reported by Farag (2010) [13]. 


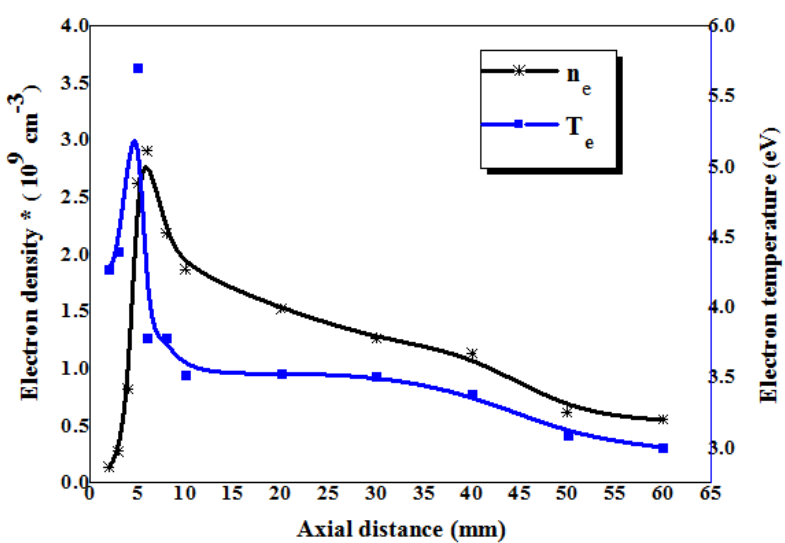

Figure (5): Axial distribution of electron (temperature and density) of $\mathrm{DC} \mathrm{O}_{2}$ plasma at gas pressure 0.5 of mbar and at a discharge power of $3.5 \mathrm{~W}$

\section{Rate}

In the present study, it is assumed that the formation of active oxygen plasma species such as $\mathrm{O}_{2}\left(a^{a^{1} \Delta_{g}}, b^{1} \Sigma_{g}^{+}\right), \mathrm{O}^{+}{ }_{2}$ and oxygen atoms $\mathrm{O}\left({ }^{3} \mathrm{P}\right.$, $\left.{ }^{1} \mathrm{D}\right)$ are resulting from direct electron impact to ground state oxygen molecules. The rate coefficients for the production of the previous active oxygen plasma species by electron impact collisions were calculated assuming the electron energy distribution is Maxwellian and fit over the electron temperature in the range 1-7 $\mathrm{V}$ [16]. According to Gudmundsson [17] the rate coefficients for electron impact ionization, dissociation and excitation for oxygen molecules in terms of electron temperature $\mathrm{T}_{\mathrm{e}}$ are given in Table (1).

The ionization rate coefficient, $\mathrm{K}_{\mathrm{iz}}$, due to direct electron impact to $\mathrm{O}_{2}$ in the ground state as a function of $\mathrm{O}_{2}$ gas pressure, is shown in Fig. (6-a). As can be seen from the figure, an increase in the $\mathrm{O}_{2}$ pressure results in a noticeable influence on the ionization rate. As the $\mathrm{O}_{2}$ gas pressure increases the ionization rate coefficient shows a decreasing trend due to the inverse relationship between gas pressure and the electron temperature.

Dissociation rate coefficients, $\mathrm{K}_{\text {diss }}{ }^{1}, \mathrm{~K}_{\text {diss }}{ }^{2}$ and $\mathrm{K}_{\text {diss }}{ }^{3}$, due to the reactions (R2,R3 and R4) for $\mathrm{O}_{2}$ plasma as a function of $\mathrm{O}_{2}$ gas pressure are illustrated in Fig. (6-b). The dissociation rate coefficients due to the three reactions showed a decreasing trend with increasing O2gas pressure. This also could be attributed to the inverse relationship between gas pressure and electron temperature. The dissociation rate coefficient due to reaction $R 3, \mathrm{~K}_{\mathrm{diss}}{ }^{2}$ is about five orders of magnitude higher than that due to reaction R2, $\mathrm{K}_{\text {diss }}{ }^{1}$. The dissociation rate coefficient due to reaction $R 4, K_{\text {diss }}{ }^{3}$ is the lowest one and the dissociation due it is of less importance.

Table (1): Electron impact ionization, dissociation and excitation rate coefficients of oxygen molecules

\begin{tabular}{|c|c|}
\hline Reaction & Rate coefficient $\left(\mathrm{m}^{3} \mathrm{~S}^{-1}\right)$ \\
\hline (R1) $e+\mathrm{O}_{2} \rightarrow \mathrm{O}_{2}^{+}+2 e$ & $k_{i z}=2.34 \times 10^{-15} T_{e}^{1.03} \exp \left(-12.29 / T_{e}\right)$ \\
\hline (R2) $e+O_{2} \rightarrow O_{2}\left(A^{3} \Sigma_{u}^{+}, A^{\prime 3} \Delta_{u}, c^{1} \Sigma_{u}^{-} \rightarrow O\left({ }^{3} P\right)+O\left({ }^{3} P\right)+e\right.$ & $k_{d i s s}^{1}=6.86 \times 10^{-15} \exp \left(-6.29 / T_{e}\right)$ \\
\hline$(\mathbf{R 3}) e+O_{2} \rightarrow O_{2}\left(B^{3} \Sigma_{u}^{-}, B^{3} \Sigma_{u}^{-}, 2^{3} \Pi_{u}\right) \rightarrow O\left({ }^{3} P\right)+O\left({ }^{1} D\right)+e$ & $k_{d i s s}^{2}=3.49 \times 10^{-14} \exp \left(-5.92 / T_{e}\right)$ \\
\hline$(\mathrm{R4}) \mathrm{e}+\mathrm{O}_{2} \rightarrow O\left({ }^{1} \mathrm{D}\right)+O\left({ }^{1} \mathrm{D}\right)+e$ & $k_{\text {diss }}^{3}=1.44 \times 10^{-16} \exp \left(-17.25 / T_{e}\right)$ \\
\hline${ }_{(\mathrm{R} 5)} e+\mathrm{O}_{2}\left(X^{3} \Sigma_{g}^{-}\right) \rightarrow O_{2}\left(a^{1} \Delta_{g}\right)+e$ & $k_{e x}^{\text {delta }}=1.37 \times 10^{-15} \exp \left(-2.14 / T_{e}\right)$ \\
\hline (26) $e+O_{2}\left(X^{3} \Sigma_{g}^{-}\right) \rightarrow O_{2}\left(b^{1} \Sigma_{g}^{+}\right)+e$ & $k_{e x}^{\text {sigma }}=3.24 \times 10^{-16} \exp \left(-2.218 / T_{e}\right)$ \\
\hline
\end{tabular}


The excitation rate coefficients of metastable oxygen molecule $\mathrm{O}_{2} \quad\left(a^{1} \Delta_{g}\right.$ and $\left.b^{1} \Sigma_{g}^{+}\right)$from ground state, $\mathrm{K}_{\mathrm{ex}}{ }^{\text {delta }}$ and $\mathrm{K}_{\mathrm{ex}}{ }^{\text {sigma }}$, due to the reactions (R5and R6) for $\mathrm{O}_{2}$ plasma as a function of $\mathrm{O}_{2}$ pressure are shown in Fig. (6-c). The excitation rate coefficients, $\mathrm{K}_{\mathrm{ex}}{ }^{\text {delta }}$ and $\mathrm{K}_{\mathrm{ex}}{ }^{\text {sigma }}$, showed a decreasing trend with increasing $\mathrm{O}_{2}$ gas pressure. Also, the values of $\mathrm{K}_{\mathrm{ex}}{ }^{\text {delta }}$ are higher than those for $K_{e x}{ }^{\text {iigma at }}$ at the same electron temperature. This could be attributed to the excitation cross section for the transition for $\mathrm{O}_{2}\left(X^{3} \Sigma_{g}^{-}\right) \rightarrow \mathrm{O}_{2}($ $\left.a^{1} \Delta_{g}\right)$ is larger than that for the transition $\mathrm{O}_{2}($ $\left.X^{3} \Sigma_{g}^{-}\right) \rightarrow \mathrm{O}_{2}\left(b^{1} \Sigma_{g}^{+}\right)$at the same temperature.

The axial profiles of ionization, dissociation and excitation rate coefficients for oxygen discharge at operating conditions as $\mathrm{O}_{2}$ gas pressure $=0.5 \mathrm{mbar}$ and applied power about $3.5 \mathrm{~W}$ are shown in Fig.(7). The axial profiles of ionization, dissociation and excitation rate coefficients are similar to that of the electron temperature (see Fig. 5). Also, it should be noted that the ionization, dissociation and excitation rate coefficients occur over a large area in the negative glow region comparing to it is in the $\mathrm{CF}$ region. These results are in good agreement with those given by Meyyappan and Kreskovesky [18] who concluded that in low pressure DC glow discharges, most of ionization and excitation occur in the negative glow region rather than in the $\mathrm{CF}$ region.

The rate production of $\mathrm{O}_{2}^{+}$, atoms $\mathrm{O}\left({ }^{3} \mathrm{P},{ }^{1} \mathrm{D}\right)$ and $\mathrm{O}_{2}\left(a^{1} \Delta_{g}, b^{1} \Sigma_{g}^{+}\right)$via the ionization, dissociation and excitation reactions, respectively was calculated by the use of the obtained values of rate coefficients $(k)$ and electron number density $\left(\mathrm{n}_{\mathrm{e}}\right)$ in the above sections according to the following expression [19]:

$$
R=n_{e} n_{g} k
$$

Where $n_{g}$ is the neutral gas density, is calculated from the pressure and temperature of the gas by using ideal gas law $\left(\mathrm{n}_{\mathrm{g}}=\mathrm{P} / \mathrm{K}_{\mathrm{B}} \mathrm{T}_{\mathrm{g}}\right)$. In the present work, $\mathrm{T}_{\mathrm{g}}$ is measured by a thermal couple and is $300 \mathrm{~K}$.

The ionization, dissociation and excitation rates for $\mathrm{O}_{2}$ plasma as a function of $\mathrm{O}_{2}$ gas pressure are illustrated in Fig. (8). It can be noticed that the behavior of the three rates with the $\mathrm{O}_{2}$ pressure is the same where the three rates shown an increasing trend with increasing $\mathrm{O}_{2}$ gas pressure. According to equation 1 , the reaction rate is directly proportional to the electron number density, the density of the neutral particles and reaction rate coefficient, so with an increasing $\mathrm{O}_{2}$ gas pressure: $\mathrm{n}_{\mathrm{e}}$ and $\mathrm{n}_{\mathrm{g}}$ increase and consequently the reaction rate increases while $T_{\mathrm{e}}$ decreases and hence the reaction rate coefficient $(\mathrm{k})$ decreases; consequently, the reaction rate decreases. Based on the present results, the effect of $n_{e}$ and $n_{g}$ is more effective than $\mathrm{k}$. Therefore, the rate of the ionization, dissociation and excitation reactions increases by increasing the $\mathrm{O}_{2}$ gas pressure. The behavior of the reaction rate with the gas pressure is similar to that reported in literature $[19,20]$.
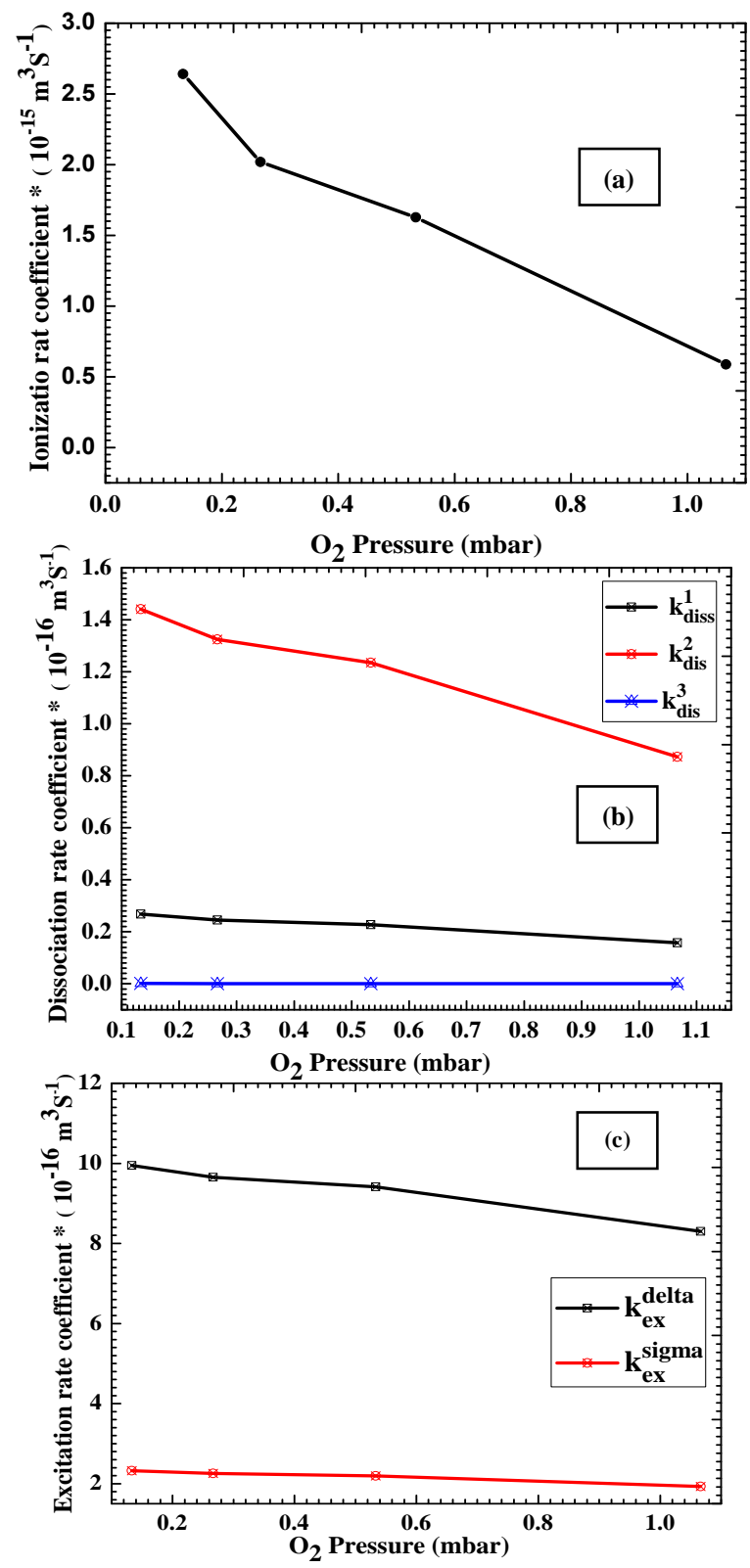

Figure (6): Variation of electron impact (a) ionization, (b) dissociation and (c) excitation rate coefficients with gas pressure in $\mathrm{DC} \mathrm{O}_{2}$ Plasma at discharge power 3.5 W 

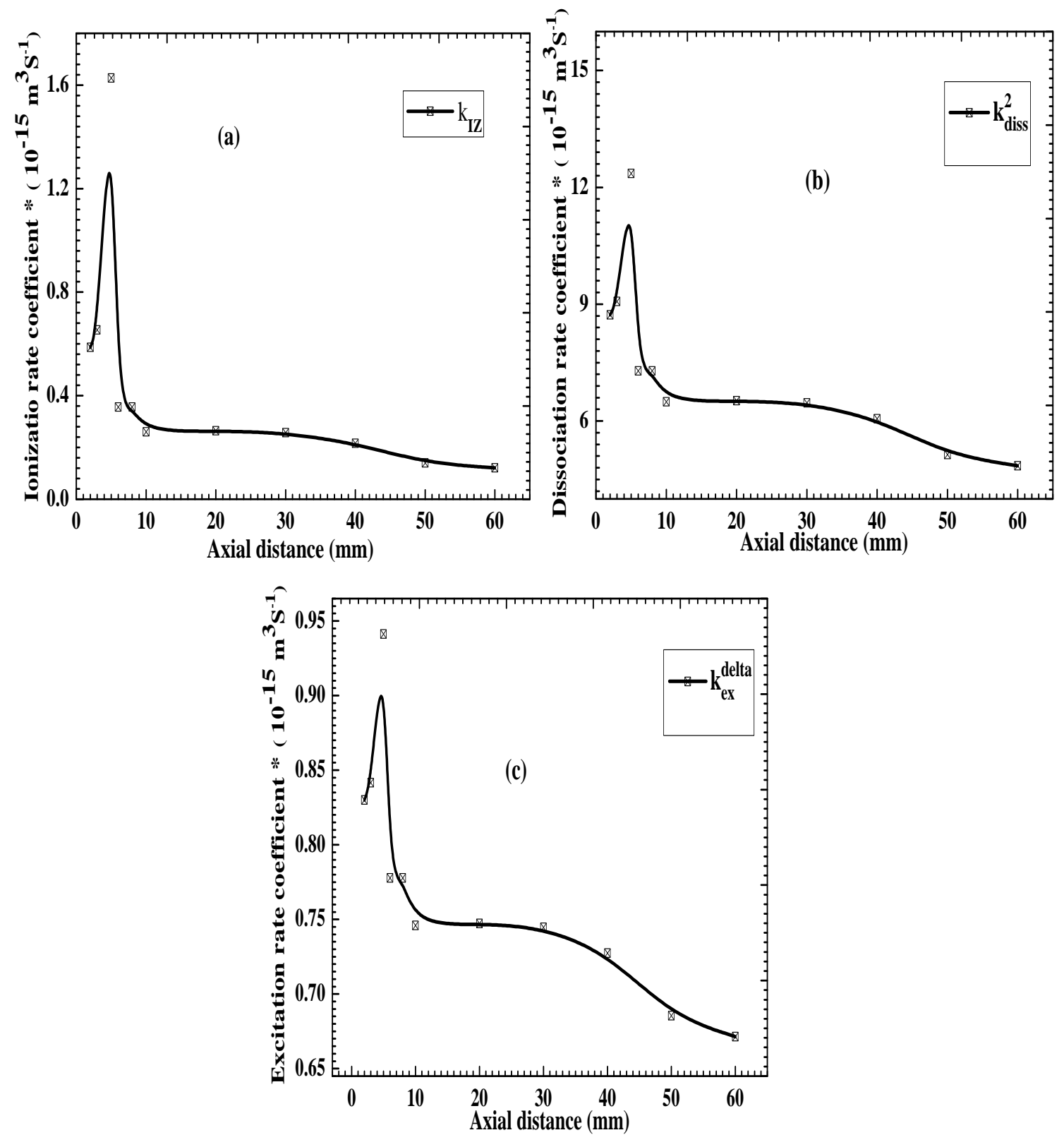

Figure (7): Axial distribution of electron impact (a) ionization, (b) dissociation and (c) excitation rate coefficients in $\mathrm{DC} \mathrm{O}_{2}$ Plasma at discharge power $3.5 \mathrm{~W}$ and $\mathrm{P}=0.5 \mathrm{mbar}$ 
The axial profiles of electron impact ionization, dissociation and excitation rates for oxygen discharge at the operating conditions of $\mathrm{O}_{2}$ gas pressure $=0.5$ mbar and applied power about 3.5 $\mathrm{W}$ are shown in Fig.(9). The axial profiles of ionization, dissociation and excitation rates are similar to that of the electron number density (see Fig. 5). It should also be noted that the ionization, dissociation and excitation rates occur over a large area in the negative glow region comparing to it is in the CF region.

\section{Conclusion}

The electrical characteristics and plasma parameters of $\mathrm{DC} \mathrm{O}_{2}$ plasma at low pressure have been investigated experimentally. The DC glow discharge plasma generated in the present investigation operate in the normal mode where the discharge current is in the range 1 to $10 \mathrm{~mA}$ and increases with increasing operating voltage. The breakdown voltage depends on the product of $(\mathrm{Pd})$. The Values of electron temperature $\left(T_{e}\right)$ and density $\left(n_{e}\right)$ which are necessary to calculate the rate coefficients and rates of electron impact ionization, dissociation and excitation have been determined by using the current-voltage characteristic curves of the single Langmuir probe. As the plasma power is constant at $3.5 \mathrm{~W}$, the $\mathrm{T}_{\mathrm{e}}$ decreased with increasing $\mathrm{O}_{2}$ gas pressure while $\mathrm{n}_{\mathrm{e}}$ increased with increasing $\mathrm{O}_{2}$ gas pressure. The rate coefficients of ionization, dissociation and excitation reactions decrease with increasing $\mathrm{O}_{2}$ gas pressure while their rates increased with increasing $\mathrm{O}_{2}$ gas pressure. The axial profiles of ionization, dissociation and excitation rate coefficients and rates have maximum at the edge of the negative glow region.

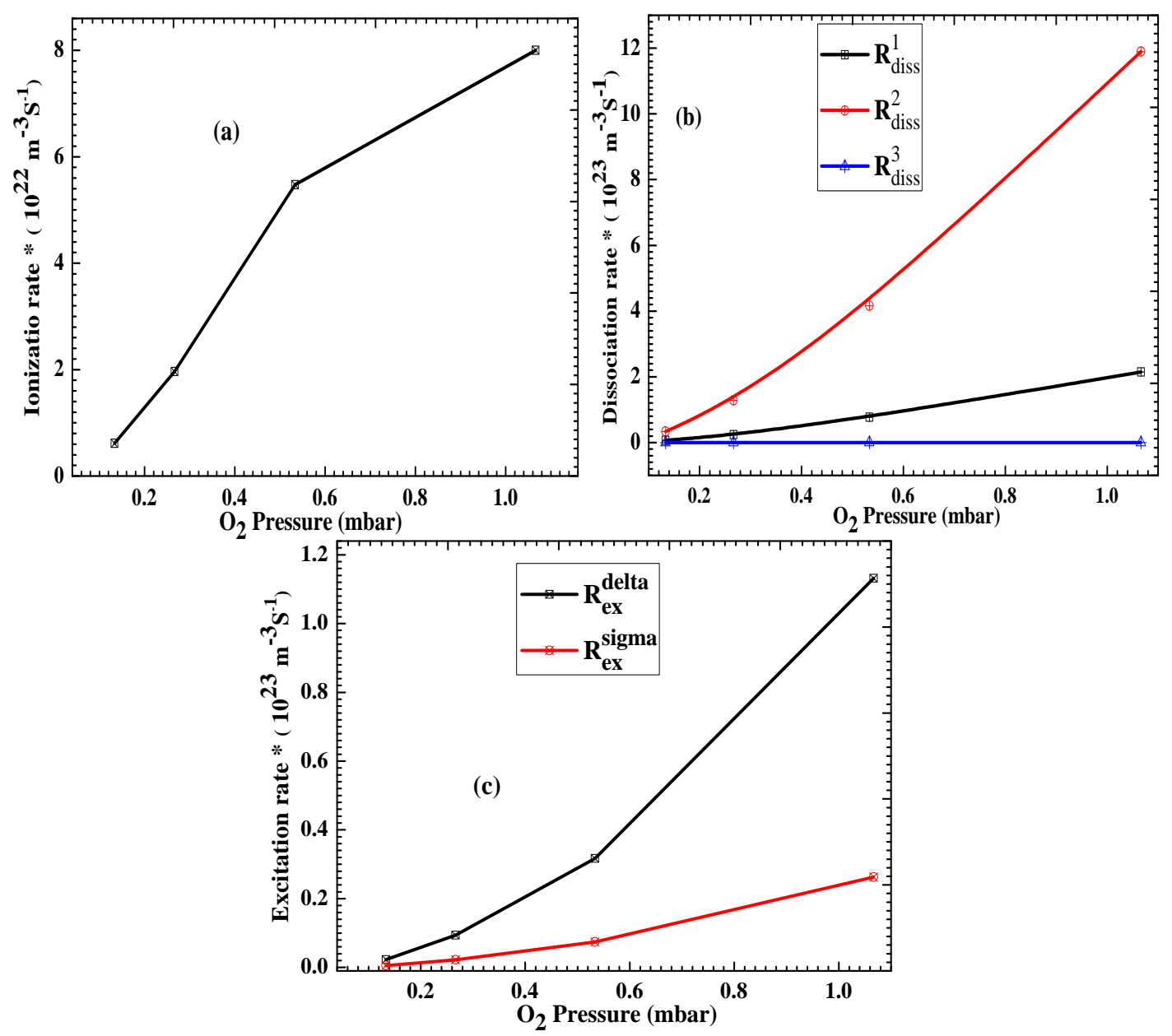

Figure (8): Variation of electron impact (a) ionization, (b) dissociation and (c) excitation rate with gas pressure in $\mathrm{DC}_{2}$ Plasma at discharge power $3.5 \mathrm{~W}$ 

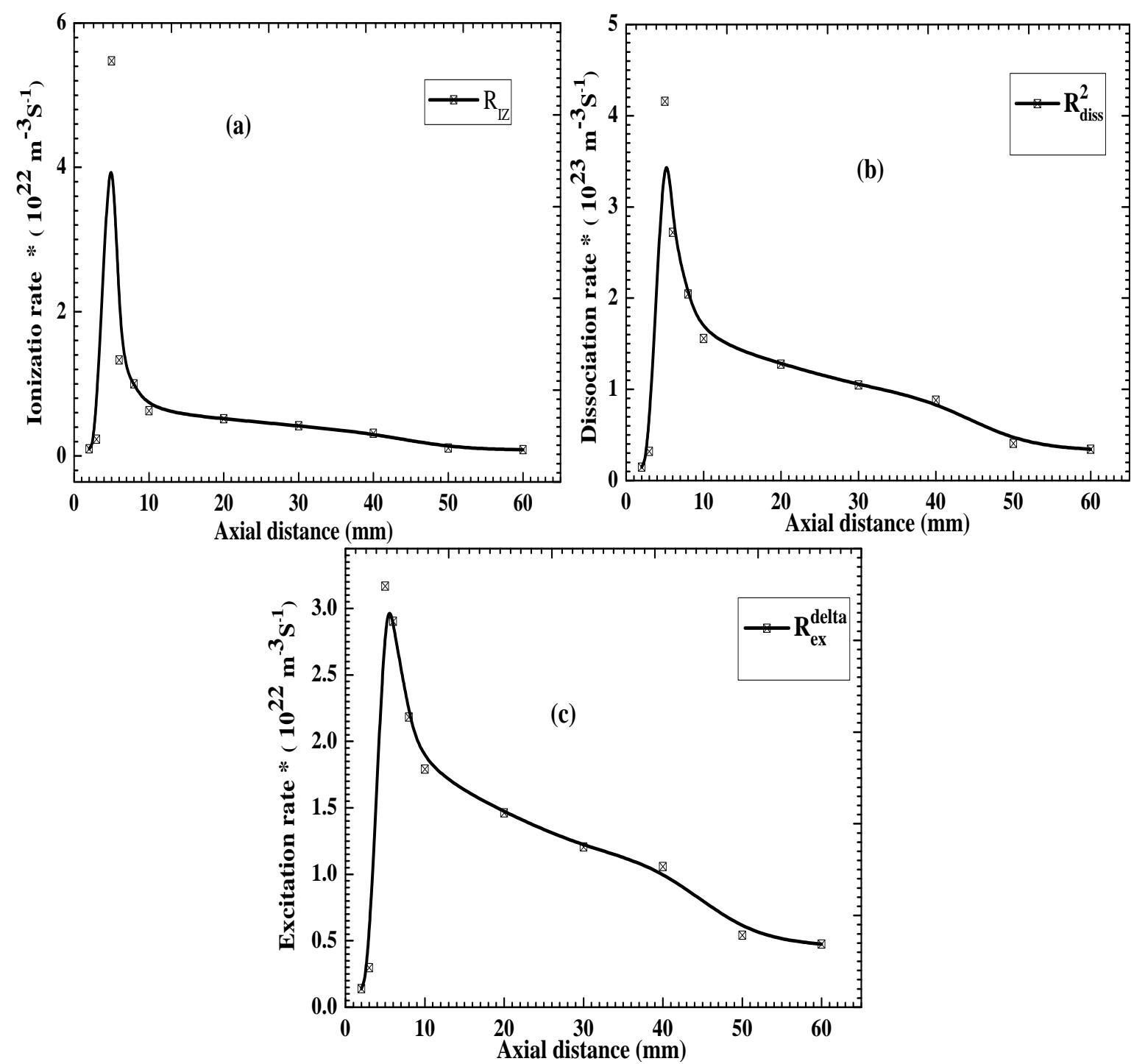

Figure (9): Axial distribution of electron impact (a) ionization, (b) dissociation and (c) excitation rate in $\mathrm{DC} \mathrm{O}_{2}$ Plasma at discharge power $3.5 \mathrm{~W}$ and $\mathrm{P}=0.5 \mathrm{mbar}$

\section{References}

1-J T Gudmundsson and E G Thorsteinsson,Oxygen discharges diluted with argon: dissociation processes, Plasma Sources Science and Technology, 2007, 16,399.

2-D A Carl, D W Hess, M A Lieberman, T D Nguyen and R Gronsky,Effects of dc bias on the kinetics and electrical properties of silicon dioxide grown in an electron cyclotron resonance plasma, Journal of Applied Physics, 1991, 70, 3301.

3-M Kitajima, H Kuroki, H Shinno and K G Nakamura, Growth of silicon oxide on silicon in the thin film region in an oxygen plasma, Solid State Communications, 1992, 83, 385.

4-A ABolshakov, B A Gruden, R. Mogul, M V V S Rao, S P Sharama, B N Khare and M Meyyappan,
Radio-frequency oxygen plasma as a sterilization source, AIAA Journal, 2004, 42, 823.

5-S Modarresi, M Soltanieh, S A Mousavi and I Shabani, Effect of low-frequency oxygen Plasma on Polysulfone membranes for $\mathrm{CO}_{2} / \mathrm{CH}_{4}$ Separation, Journal of Applied Polymer Science, 2012, 124, E199.

6-SK Jong andHK Gon, Characterization of an Oxygen Plasma By Using a Langmuir Probe in a Inductively Coupled Plasma, Journal of the Korean Physical Society,2001,38, 259

7-A Bogaerts, 1996, PhD thesis, University of Antwerp,Belgium.

8-K Kutasi, V Guerra, P A Sa, Active species downstream of an $\mathrm{Ar}-\mathrm{O}_{2}$ surface-wave microwave discharge for biomedicine, surface treatment and 
nanostructuring, Plasma Sources Science and Technology, 2011, 20,035006.

9-D Maric, M Savic, J Sivos, N Skoro, M R Radjenovic, G Malovic, and $\mathrm{Z} \mathrm{Lj}$. Petrovi, Gas breakdown and secondary electron yields, European Physical Journal D, 2014, 68, 1.

10-M K Khalaf, I R Agooi and S H Abd Muslim, Electrical Characteristics and plasma diagnostics of $\left(\mathrm{Ar} / \mathrm{O}_{2}\right)$ gas mixture glow discharge, International Journal of Application or Innovation in Engineering and management, 2014, 3, 113.

11-F Araghi and D Dorranian, Effect of negative ions on the characteristics of plasma in a cylindrical discharge, Journal of Theoretical and Applied Physics, 2013, 7:41, 1-8.

12-K Yasserian, M Ghornneviss, M Aslaninejad, Langmuir probe measurements in a cylindrical magnetron discharge in the presence of $\mathrm{Ar} / \mathrm{O} 2$, Japanese Journal of Applied Physics, 2009, 48, 036001.

13-O F Farag, Msc thesis, 2010, Zagazig University, Egypt.

14-M M Mansour, N M El-Sayed, O F Farag and M H Elghazaly; Effect of $\mathrm{He}$ and $\mathrm{Ar}$ addition on $\mathrm{N}_{2}$ glow discharge characteristics and plasma diagnostics, Arab Journal of Nuclear Science and Applications, 2013, 46, 116.
15-M M Mansour and O F Farag, Experimental study of the influence of nitrogen admixture on characteristics of DC glow discharge in He gas, Der ChemicaSinica, 2013, 4,94.

16-J T Gudmundsson, A M Marakhtanov, K K Patel, V $\mathrm{P}$ Gopinath and $\mathrm{M}$ A Lieberman, On the plasma parameters of a planar inductive oxygen discharge, Journal of Physics D: Applied Physics, 2000, 33, 1323.

17-J T Gudmundsson, A critical review of the reaction set for a low pressure oxygen processing discharge, Department of electrical and computer engineering, university of Iceland, Iceland, (2004).

18-M Meyyappan and J P Kreskovesky, Glow discharge simulation through solutions to the moments of the Boltzmann transport equation, Journal of Applied Physics, 1990, 68, 1506-12.

19-J T Gudmundsson, I G Kouznestov, K K Patel M A Lieberman, Electronegativity of Low-Pressure HighDensity Oxygen Discharges, Journal of Physics D: Applied Physics, 2001, 34, 1100.

20-M H Elghazaly and S Solyman, Electron impact ionization and excitation rate coefficients in the negative glow region of a glow discharge, Journal of Quantitative Spectroscopy and Radiative transfer, 2007, 103, 260. 